\title{
Spatial variations in the star formation history of the Large Magellanic Cloud
}

\author{
Carme Gallart ${ }^{1}$, Ingrid Meschin ${ }^{1}$, Antonio Aparicio ${ }^{1}$, \\ Peter B. Stetson ${ }^{2}$ and Sebastián L. Hidalgo ${ }^{1}$ \\ ${ }^{1}$ Instituto de Astrofísica de Canarias. 38200 La Laguna. Tenerife, Spain \\ email:carme, imeschin, antapaj, shidalgo@iac.es \\ ${ }^{2}$ Herzberg Institute of Astrophysics, National Research Council, Victoria, BC, \\ Canada V9E 2E7 \\ email: Peter.Stetson@nrc-cnrc.gc.ca
}

\begin{abstract}
Based on the quantitative analysis of a set of wide-field color-magnitude diagrams reaching the old main sequence-turnoffs, we present new LMC star-formation histories, and their variation with galactocentric distance. Some coherent features are found, together with systematic variations of the star-formation history among the three fields analyzed. We find two main episodes of star formation in all three fields, from 1 to 4 and 7 to $13 \mathrm{Gyr}$ ago, with relatively low star formation around $\simeq 4-7$ Gyr ago. The youngest age in each field gradually increases with galactocentric radius; in the innermost field, LMC 0514-6503, an additional star formation event younger than 1 Gyr is detected, with star formation declining, however, in the last $\simeq 200 \mathrm{Myr}$. The population is found to be older on average toward the outer part of the galaxy, although star formation in all fields seems to have started around 13 Gyr ago.
\end{abstract}

Keywords. galaxies: evolution, galaxies: formation, galaxies: individual (LMC), Magellanic Clouds

\section{Introduction}

Modern hydrodynamical simulations of galaxy formation and evolution are beginning to be able to predict both temporally and spatially resolved structure in galaxies (e.g., Abadi et al. 2003; Governato et al. 2007; Roškar et al. 2008), and thus, it is timely to offer detailed observational counterparts for comparison with the models.

From the observational side, late-type spiral and irregular galaxies are found to become on average redder with increasing distance from the center (e.g., Taylor et al. 2005). On another hand, Local Group dwarf irregular galaxies show stellar population gradients such that young populations disappear in the outer part (e.g., Bernard et al. 2007). This has sometimes been interpreted (Minniti \& Zijlstra 1996) as the presence of an old stellar halo in these galaxies. However, with typical groundbased observations of galaxies at $\simeq 1 \mathrm{Mpc}$, such as those of Minniti \& Zijlstra, it is not possible to ascertain the actual age composition, after the last few hundred Myr, as a function of position. Carbon stars indicate the presence of intermediate-age populations at large radius. For example, in IC 1613 Albert et al. (2000) found $24 \mathrm{C}$ stars beyond $\mathrm{R} \simeq 10^{\prime}$, where the color - magnitude diagrams (CMD) of Bernard et al. (2007) show few or no stars on the young main sequence (MS).

A quantitative determination of the star-formation history ( $\mathrm{SFH}$ ) as a function of radius can be obtained from CMDs reaching down to the oldest MS turnoffs (Gallart et al. 1999; Gallart et al. 1999, 2005; Cole et al. 2007). These can be obtained using ground-based facilities for all the Milky Way satellites. In addition, an important fraction of the Local Group galaxies can be observed down to the same absolute magnitude limit 
using the ACS on board the HST (e.g., Bernard et al. 2008; Cole et al. 2007; Brown et al. 2007).

Thanks to their proximity, the Magellanic Clouds are in principle ideal candidates for detailed studies of their SFHs, and can help to shed some light on the actual origin of the stellar-population gradients observed in late-type spiral and irregular galaxies. Their huge size in the sky, however, makes it challenging to observe areas large enough to contain representative samples of stars. Most studies to date that do reach the oldest MS turnoffs of field LMC stars have used the WFPC2 on the HST to observe relatively small fields in the bar or at relatively small galactocentric distances (single WFPC2 fields in the case of Holtzman et al. 1999; mosaics of a few fields in the case of Smecker-Hane et al. 2002). In other cases, the field stars have been studied in images taken for the purpose of observing clusters (e.g., Olsen 1999; Javiel et al. 2005). The situation is similar in the case of the SMC (Dolphin et al. 2001; McCumber et al. 2005; Chiosi \& Vallenari 2007; Noël et al. 2007).

In this paper, we will discuss some new results on the SFH of the LMC and its variation as a function of galactocentric distance, based on a quantitative analysis of a set of four wide-field CMDs reaching the old MS turnoffs, and ranging from $2.3^{\circ}$ to $7.1^{\circ}$. We will compare these results with others on late-type and irregular galaxies in order to shed light on some of the issues raised above.

\section{The data}

We obtained V- and I-band images of four LMC fields with the Mosaic II camera on the CTIO Blanco $4 \mathrm{~m}$ telescope in December 1999 and January 2001. Fields were chosen to span a range of galactocentric distances, from $\simeq 2.3^{\circ}$ to $7.1^{\circ}(2.0$ to $6.2 \mathrm{kpc})$ northward from the kinematic center of the LMC. We will name the fields according to their RA and DEC (J2000.0) as LMC 0512-6648, LMC 0514-6503, LMC 0513-6333 and LMC 0513-6159, in order of increasing galactocentric distance. Profile-fitting photometry was obtained with the DAOPHOT/ALLFRAME suite of codes (Stetson 1994) and calibrated to the standard system using observations of several Landolt (1992) fields obtained in the same runs. Finally, a large number of artificial star tests were performed in each frame following the procedure described in Gallart et al. (1999); these are used both to derive completeness factors and to model photometric errors in the synthetic CMDs.

\section{The star formation history and its spatial variations}

Figure 1 shows the $M_{I} \lesssim 4$ mag CMDs of the four LMC fields, with isochrones from the overshooting set of the BaSTI library (Pietrinferni et al. 2004) $\dagger$ superimposed. The metallicities have been chosen to approximately reproduce the common chemical enrichment law for the same fields derived by Carrera et al. (2008), using Ca II triplet spectroscopy. Note how well this combination of ages and metallicities reproduces the position and shape of the RGB. The number of stars observed with good quality photometry down to $M_{I} \lesssim 4$ in each field - in order of increasing galactocentric distances — are 300000, 214000, 86000 and 39000 respectively. All the CMDs reach the oldest MS turnoff $\left(M_{I} \simeq 3.0 \mathrm{mag}\right)$ with good photometric precision and completeness fractions over $75 \%$ (except for the innermost field, in which crowding is very severe). The two innermost fields show CMDs with a prominent, bright MS and a well populated red clump

$\dagger$ The new 2008 version of the BaSTI stellar evolution models (see http://www.oa-teramo.inaf.it/BASTI) is used through the paper. This set shows a much better agreement with other stellar evolution models than the older one. 


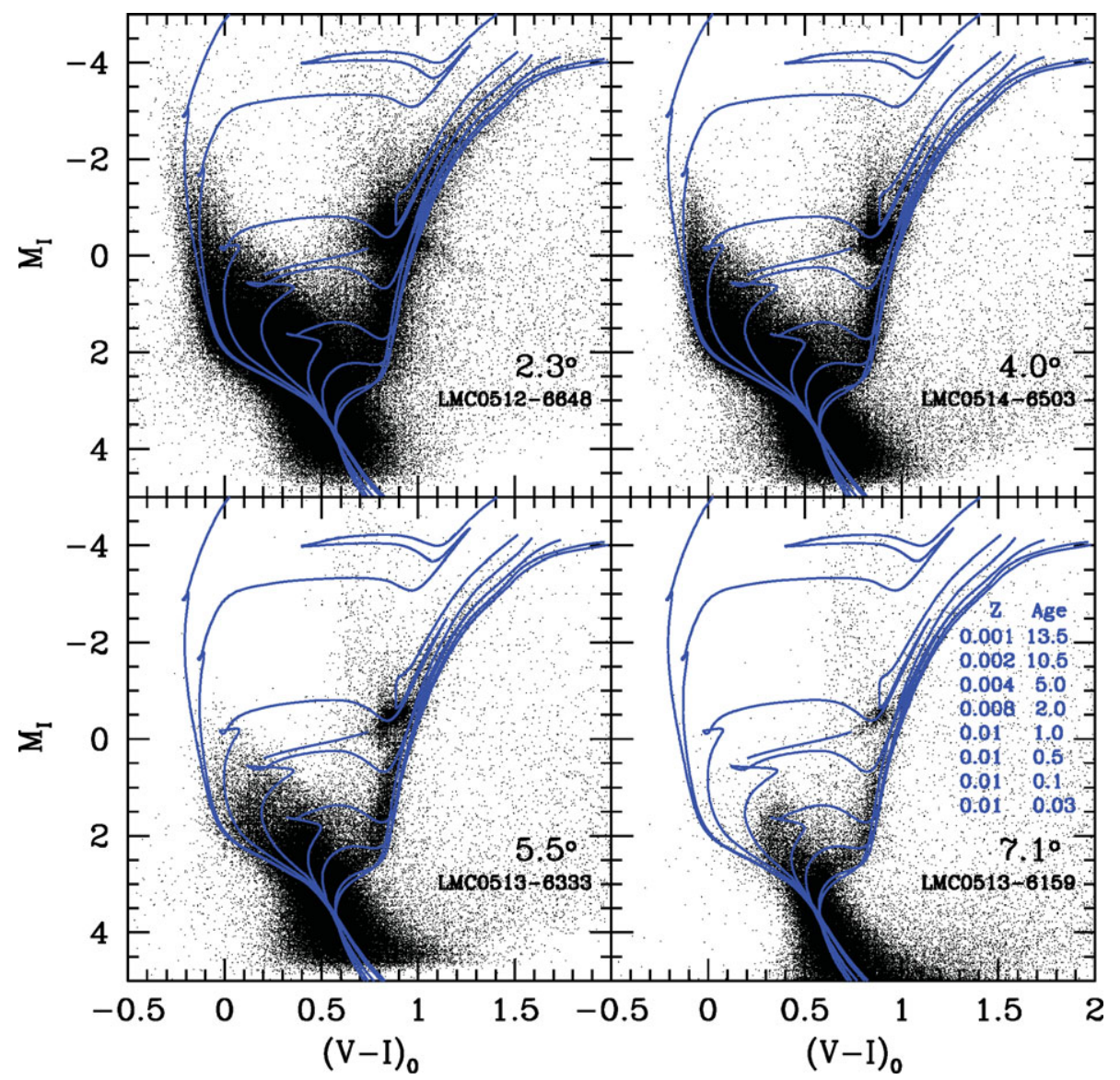

Figure 1. $\left[(V-I)_{0}, M_{I}\right]$ CMDs for the four fields. Isochrones with ages and metallicities as labelled, and a zero-age horizontal-branch of $Z=0.001$ by Pietrinferni et al. (2004) have been superimposed. A distance modulus of $(m-M)_{0}=18.5$ and $E(B-V)=0.10,0.05,0.037$ and 0.026 magnitudes, respectively, have been assumed.

typical of a population which has had ongoing star formation from $\simeq 13$ Gyr ago to the present time. The two outermost fields clearly show a fainter MS termination, indicating truncated or sharply decreasing star formation in the last few hundred Myr or few Gyr, respectively (see below). No extended horizontal branch is observed in any of the fields, but all fields host a number of stars redder than the RGB tip (and redder than the color interval shown in the figure), which are candidate AGB stars.

Gallart et al. (2008) discussed the LMC disk stellar populations and their gradients, based on a comparison of the observed CMD with the BaSTI isochrones, as well as a comparison of the observed color functions (CF) with synthetic CFs. They concluded the following: (a) the area around the old MS turnoff is well populated in all four CMDs, indicating that star formation started at about the same time, $\simeq 13 \mathrm{Gyr}$ ago, in all four fields, or that old stars have been able to migrate out to the outermost galactocentric radii observed here; (b) the bulk of the star formation may be truncated in the outermost fields (LMC 0513-6159 and LMC 0513-6333) at ages $\simeq 1.5$ and 0.8 Gyr respectively; and (c) there are indications of enhanced star formation (as compared to a constant star formation rate) in the innermost fields LMC 0514-6503 and LMC 0512-6648 in the same time range, likely having started even earlier, $\sim 4$ Gyr ago. Enhanced star formation 


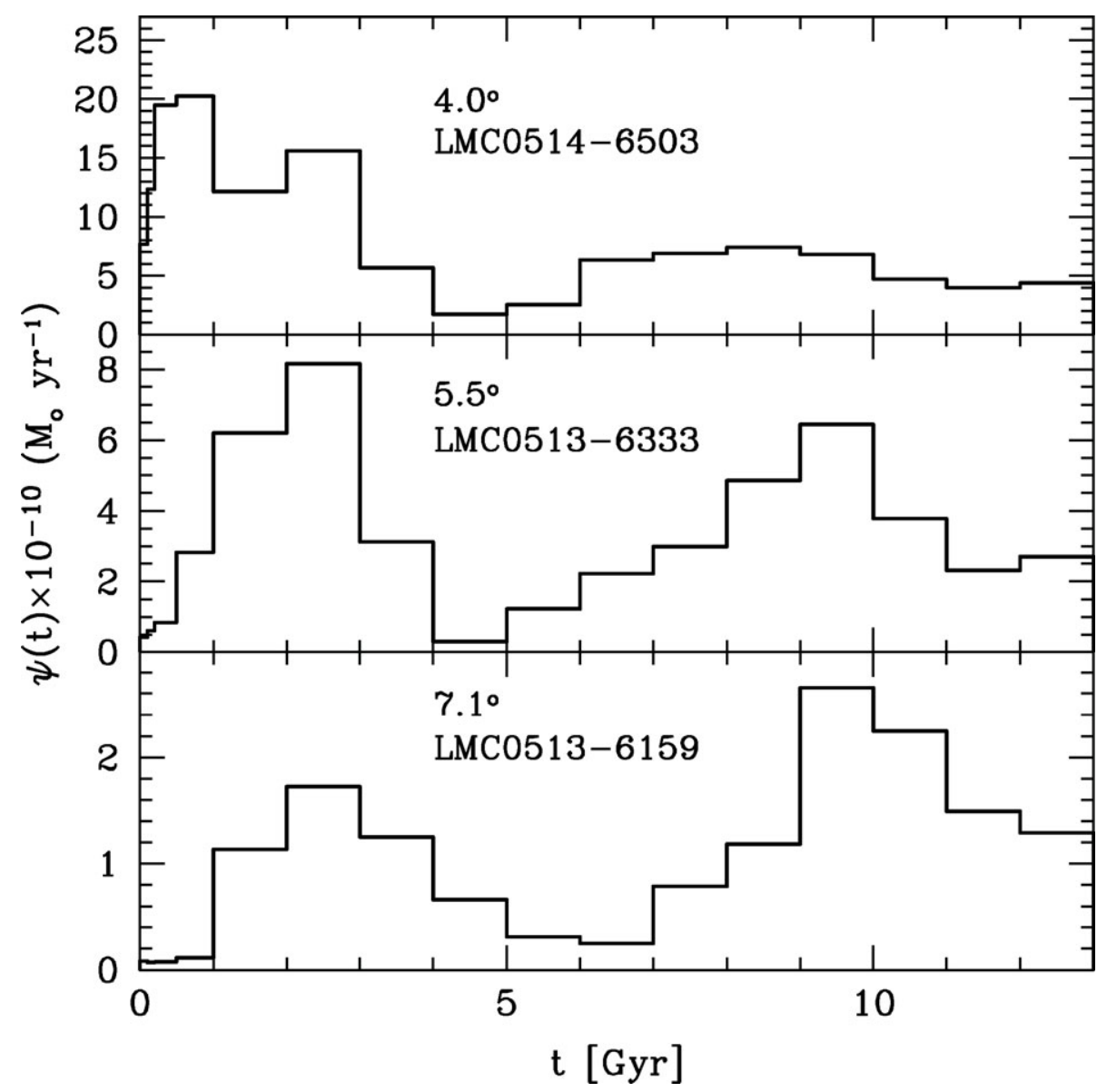

Figure 2. SFH of the three outermost fields, projected on the $(\psi(t), t)$ plane.

starting around 4 Gyr ago possibly extended also to field LMC 0513-6333; however in this case it would have been followed by the truncation discussed above.

To obtain a quantitative derivation of the $\mathrm{SFH}$ in the observed fields, we have used the IAC-STAR, IAC-POP and MINNIAC codes (Aparicio \& Gallart 2004; Aparicio \& Hidalgo 2008; Hidalgo et al. 2008, in preparation) to compute synthetic CMDs and compare the distribution of stars in the observed and synthetic CMDs, obtaining the best solution through $\chi^{2}$ minimization (see the references above and Meschin et al. 2008, in these proceedings, for details on the synthetic CMD technique and our particular implementation of it). Here, and in Meschin et al. (2008), we show preliminary solutions for the three outermost fields, LMC 0513-6159, LMC 0513-6333 and LMC 0514-6503. We are in the process of testing the robustness of the solutions and their dependence on variations of some of the input parameters, such as the binary fraction or the IMF, or on the distance modulus and reddening adopted. We believe, however, that the present solutions provide a reasonable description of the main features characterizing the LMC SFH at different galactocentric radii. We have assumed the Kroupa (2001) IMF, a binary fraction of 0.4 with mass ratios $q \geqslant 0.5$, a distance modulus $(m-M)_{0}=18.5$ and reddenings $E(B-V)=0.05,0.037$ and 0.026 magnitudes, for the innermost to the outermost field, respectively. The BaSTI stellar evolution library has been used as input to IAC-STAR to compute the synthetic CMDs. Figure 2 shows the projection of the SFHs 
on the $(\psi(t), t)$ plane (see the poster by Meschin et al. 2008 for a 3-D representation of the $\mathrm{SFH}$ on the $(\psi(t, z), t, z)$ space).

Some coherent features, together with systematic variations of the SFH among fields, are noticeable in Figure 2. In all fields, local maxima of $\psi(t)$ are found around $\simeq 3-4$ and 8-10 Gyr ago, with relatively low star formation activity around $\simeq 4-7$ Gyr ago. The ratio between the amount of star formation younger and older than the age of the minimun $\psi(t)$ decreases toward the outer part of the galaxy, thus the population is on average older there. Star formation in all fields seems to have started around 13 Gyr ago. These features are consistent with the age distribution of star clusters in the LMC. The trends regarding the young and young intermediate-age SFH that have been found through the comparison with isochrones and the use of the CF (Gallart et al. 2008; see above) are fully confirmed with the quantitative analysis presented in this paper: the youngest age in each field gradually increases with galactocentric radius, from $2.3^{\circ}$ to $7.1^{\circ}$. In particular, in the field at $4.0^{\circ}$ an additional star formation event younger than 1 Gyr is detected, with star formation declining, however, in the last $\simeq 200 \mathrm{Myr}$.

\section{Discussion}

Since no plausible galaxy-formation theory predicts positive metallicity gradients, Taylor et al. (2005) offered two possible explanations for the fact that the late-type galaxies in their sample become redder outwards: a change in the mean age of stellar population, or a change in the dust characteristics. The present work shows that the youngest, bluest stars are progressively missing outwards in the LMC disk, and that the fraction of old and intermediate-age population relative to the younger population increases at increasing galactocentric radius. Therefore, a reddening of the intrinsic integrated color is expected. A similar trend in found in the dwarf galaxies observed in the framework of the LCID project (Gallart et al. 2007), and in particular in the case of IC 1613 and Phoenix: the population gets gradually older outwards, though a sizable amount of intermediateage population is found at all radii observed (so far). Other irregular galaxies (and M33) are not close enough for such a detailed picture to be obtained, but shallower CMDs hint at a similar situation. In addition, in the case of the LMC, the surface brightness profile remains exponential to the largest galactocentric radius observed in this work, and shows no evidence of disk truncation. Combining the information on surface brightness and stellar population, Gallart et al. (2004) concluded that the LMC disk extends - and dominates over a possible stellar halo - out to a distance of at least $6 \mathrm{kpc}$.

The age gradient in the youngest LMC population is correlated with the H I column density as measured by Staveley-Smith et al. (2003): the two innermost fields are located at $\simeq 0.7 \mathrm{kpc}$ on either side of $R_{H \alpha}, \mathrm{LMC} 0512-6648$ on the local maximum of the azimuthally averaged $\mathrm{H}$ I column density with $\simeq 1.63 \times 10^{21} \mathrm{~cm}^{-2}$ (close to the $\mathrm{H} \mathrm{I}$ threshold for star formation; Skillman 1987) and LMC 0514-6503 where the azimuthally averaged H I column density is only $\simeq 5 \times 10^{20} \mathrm{~cm}^{-2}$. Finally, the two outermost fields are close to the H I radius considered by Staveley-Smith et al. to be at an H I density of $10^{20} \mathrm{~cm}^{-2}$. The outermost field, LMC 0513-6159, is approximately halfway to the tidal radius (van der Marel et al. 2002). If the youngest stars in each field were formed in situ, in the LMC we are observing an outside-in quenching of the star formation at recent times $(\simeq 1.5 \mathrm{Gyr}$ ), possibly implying a decrease in size of the H I disk able to form stars. Alternatively, star formation may have been confined to the central $\simeq 3-4 \mathrm{kpc}$, where gas resides (or is accreted to), and stars then migrate outwards (e.g., Roškar et al. 2008). In fact, it is expected that both star-formation sites and stars migrate across the LMC 
disk due to tidal interactions with the Milky Way and the SMC (e.g., Bekki \& Chiba 2005). Of course, a combination of the two scenarios is also possible.

\section{Acknowledgements}

C.G., A.A., I.M. \& S.L.H acknowledge the support from the IAC and the Spanish MEC (AYA2004-06343).

\section{References}

Abadi, M. G., Navarro, J. F., Steinmetz, M., \& Eke, V. R. 2003, ApJ, 591, 499

Albert, L., Demers, S., \& Kunkel, W. E. 2000, AJ, 119, 2780

Aparicio, A. \& Gallart, C. 2004, AJ, 128, 1465

Aparicio, A. \& Hidalgo, S. L. 2008, AJ, submitted

Bekki, K. \& Chiba, M. 2005, MNRAS, 356, 680

Bernard, E. J., Aparicio, A., Gallart, C., Padilla-Torres, C. P., \& Panniello, M. 2007, AJ, 134, 1124

Bernard, E. J., Gallart, C., \& Monelli, M., et al. 2008, ApJ, 678, L21

Brown, T. M., Smith, E., \& Ferguson, H. C., et al. 2007, ApJ, 658, L95

Carrera, R., Gallart, C., Hardy, E., Aparicio, A., \& Zinn, R. 2008, AJ, 135, 836

Chiosi, E. \& Vallenari, A. 2007, A\& A, 466, 165

Cole, A. A., Skillman, E. D., Tolstoy, E., et al. 2007, ApJ, 659, L17

Dolphin, A. E., Walker, A. R., Hodge, P. W., Mateo, M., Olszewski, E. W., Schommer, R. A., \& Suntzeff, N. B. 2001, ApJ, 562, 303

Gallart, C., Freedman, W. L., Aparicio, A., Bertelli, G., \& Chiosi, C. 1999, AJ, 118, 2245

Gallart, C., Stetson, P. B., Hardy, E., Pont, F., \& Zinn, R. 2004, ApJ, 614, L109

Gallart, C., Zoccali, M., \& Aparicio, A. 2005, ARAA, 43, 387

Gallart, C. and the LCID Team. 2007, in A. Vazdekis \& R. F. Peletier (eds.), Stellar Populations as Building Blocks of Galaxies, Proc. IAU Symp. No. 241 (San Francisco: ASP), p. 290

Gallart, C., Stetson, P. B., Meschin, I. P., Pont, F., \& Hardy, E. 2008, ApJ, 682, 89

Governato, F., Willman, B., Mayer, L., Brooks, A., Stinson, G., Valenzuela, O., Wadsley, J., \& Quinn, T. 2007, MNRAS, 374, 1479

Holtzman, J. A., Gallagher, J. S., III, \& Cole, A. A., et al. 1999, AJ, 118, 2262

Javiel, S. C., Santiago, B. X., \& Kerber, L. O. 2005, A\&A, 431, 73

Kroupa, P. 2001, MNRAS, 322, 231

Landolt, A. U. 1992, AJ, 104, 340

McCumber, M. P., Garnett, D, R., \& Dufour, R. J. 2005, AJ, 130, 1083

Minniti, D. \& Zijlstra, A. 1996, ApJ, 467, L13

Noël, N., Gallart, C., Costa, E., \& Méndez, R. 2007, AJ, 133, 2037

Olsen, K. A. G. 1999, AJ, 117, 2244

Pietrinferni, A., Cassisi, S., Salaris, M., \& Castelli, F. 2004, ApJ, 612, 168

Roškar, R., Debattista, V. P., Stinson, G. S., Quinn, T. R., Kaufmann, T., \& Wadsley, J. 2008, $A p J, 675, \mathrm{~L} 65$

Skillman, E. 1987, in C. J. Lonsdale Persson (ed.), Star Formation in Galaxies (NASA CP-2466; Washington: NASA), p. 263

Smecker-Hane, T. A., Cole, A. A., Gallagher, J. S., III, \& Stetson, P. B. 2002, ApJ, 566, 239

Staveley-Smith, L., Kim, S., Calabretta, M. R., Haynes, R. F., \& Kesteven, M. J. 2003, MNRAS, 339,87

Stetson, P. B. 1994, PASP, 106, 250

Taylor, V. A., Jansen, R. A., Windhorst, R. A., Odewahn, S. C., \& Hibbard, J. E. 2005, ApJ, 630,784

van der Marel, R. P., Alves, D. R., Hardy, E., \& Suntzeff, N. B. 2002, AJ, 124, 2639 